\section{IDDF2021-ABS-0202 CROTONYLATION OF SEPT2 PROTEIN PREDICTS POOR PROGNOSIS IN HEPATOCELLULAR CARCINOMA}

Xinyue Zhang*, Ning Zhang, Lixia Xu, Xiaoxing Li. Institute of Precision Medicine, The First Affiliated Hospital, Sun Yat-sen University

\subsection{6/gutjnl-2021-IDDF.10}

Background The tumor metastasis and invasion are restricted in the therapy of Hepatocellular carcinoma (HCC). Lysine crotonylation (Kcr) is a new posttranslational modification (PTM) found in 2011. But the function of $\mathrm{Kcr}$ in proteins is still unclear. In this study, we explored the role of crotonylome in metastasis of HCC.

Methods Kcr level of proteins in the cell lines MHCC97H (high invasive) and MHCC97L (low invasive) were detected by stable isotope labeling with amino acids in cell culture (SILAC). The differentially crotonylated proteins of two cell lines were selected by bioinformatics analysis. Kcr of proteins was confirmed by immunoprecipitation and followed by western bolt. The function in metastasis of Kcr was examined by in vivo and in vitro assay. The clinical significance of protein Kcr in HCC was examined by IHC assay.

Results By using SILAC, we found the crotonylation was higher in high invasive cells, and analysis showed that there are 199 up-crotonylated and 46 down-crotonylated sites (Fold change $>1.5, \mathrm{P}<0.05)$ in MHCC $97 \mathrm{H}$. We found crotonylation could facilitate cell invasion by increasing the substrate of crotonylation (NACR) in HCC cells. After normalized by protein levels, there are 10 down-crotonylated sites and 20 upcrotonylated sites (Fold change $>1.5, \mathrm{P}<0.05$ ) in MHCC97H. We selected SEPT2, which had the highest level of $\mathrm{Kcr}$ in those 3 proteins between $\mathrm{MHCC} 97 \mathrm{H}$ and MHCC97L, to perform further functional assays. After screening from known decrotonylases, we found SIRT2 decrotonylated SEPT2. SILAC showed SEPT2 was hyper-crotonylated at lysine 74 (K74) and 318 (K318) in high invasive cells. A single mutation at K74 (K74R) could impair the GTPase activity of SEPT2 and inhibit invasion and metastasis of HCC cells in vitro and in vivo. IHC assay in 120 cases HCC species showed that the overall survival and disease-free survival were lower in patients with a higher level of SEPT2-74cr.

Conclusions In this study, we firstly explored the role of $\mathrm{Kcr}$ on non-histone protein and therefore regulating metastasis and invasion of cancer. SEPT2, which is hyper-crotonylated in high invasive cells, can promote metastasis and invasion in
HCC, and may be utilized as a potential prognostic predictor of HCC.

\section{IDDF2021-ABS-0205 AKKERMANSIA VIABLE BACTERIA IMPROVES LIVER STEATOSIS INDUCED BY HIGH-FAT DIET RELATING TO THE REGULATION OF GUT MICROBIOTA IN C57BL/6J MICE}

Chuangyu Cao, Diwen Shou*, Haoming Xu, Hongli Huang, Yu Xia, Qing Mei, Ying Quan, Hanqing Chen, Chong Zhao, Wenjuan Tang, Huiting Chen, Yongjian Zhou. Department of Gastroenterology and Hepatology, Guangzhou Digestive Disease Center, Guangzhou First People's Hospital, School of Medicine, South China University of Technology, Guangzhou, China

\subsection{6/gutjnl-2021-IDDF.11}

Background Akkermansia was decreased in non-alcoholic fatty liver disease (NAFLD). Our aim was to explore the effect and mechanism of Akkermansia on NAFLD mice.

Methods Twenty-five C57BL/6J mice were divided into 5 groups (CD, HFD, HFD+ LAKK, HFD+ PAKK, HFD+ supernatant). The NAFLD mouse model was induced by a high-fat diet for 16 weeks. The mice were subsequently gavaged with different substances for 4 weeks (PBS solution for CD and HFD group, Akkermansia viable for HFD+ LAKK group, pasteurized Akkermansia for HFD+ PAKK group cultured supernatant solution for HFD+ supernatant group, 0.2 $\mathrm{mL} / \mathrm{mouse} /$ day, $2 * 10^{9} \mathrm{CFU} / \mathrm{mL}$ ). After treatment, liver steatosis was evaluated by HE staining and NAS score. Serum alanine aminotransferase (ALT), triglyceride (TG), fasting serum glucose (FBG) and insulin resistance index (HOMA-IR) were detected. The intestinal content of mice was collected for microbiota analysis by $16 \mathrm{~s}$ amplicon sequencing.

Results Compared with HFD group, liver steatosis in HFD + LAKK group was significantly improved, and the serum ALT, TG, FBG and HOMA-IR were significantly decreased $(P<0.05)$ (IDDF2021-ABS-0201 Figure 1a. Akkermansia viable improved liver steatosis; Hepatic HE staining of mice) (IDDF2021-ABS-0201 Figure 1b. Akkermansia viable improved liver steatosis; NAS score of mice $(* P<0.01))($ IDDF2021ABS-0205 Figure 1c. Akkermansia viable improved liver steatosis; serum levels of ALT $(* P<0.05)$ ) (IDDF2021-ABS-0205 Figure 1d. Akkermansia viable improved liver steatosis; serum levels of TG $(* P<0.01))$ (IDDF2021-ABS-0205 Figure 1e. Akkermansia viable improved liver steatosis; serum levels of
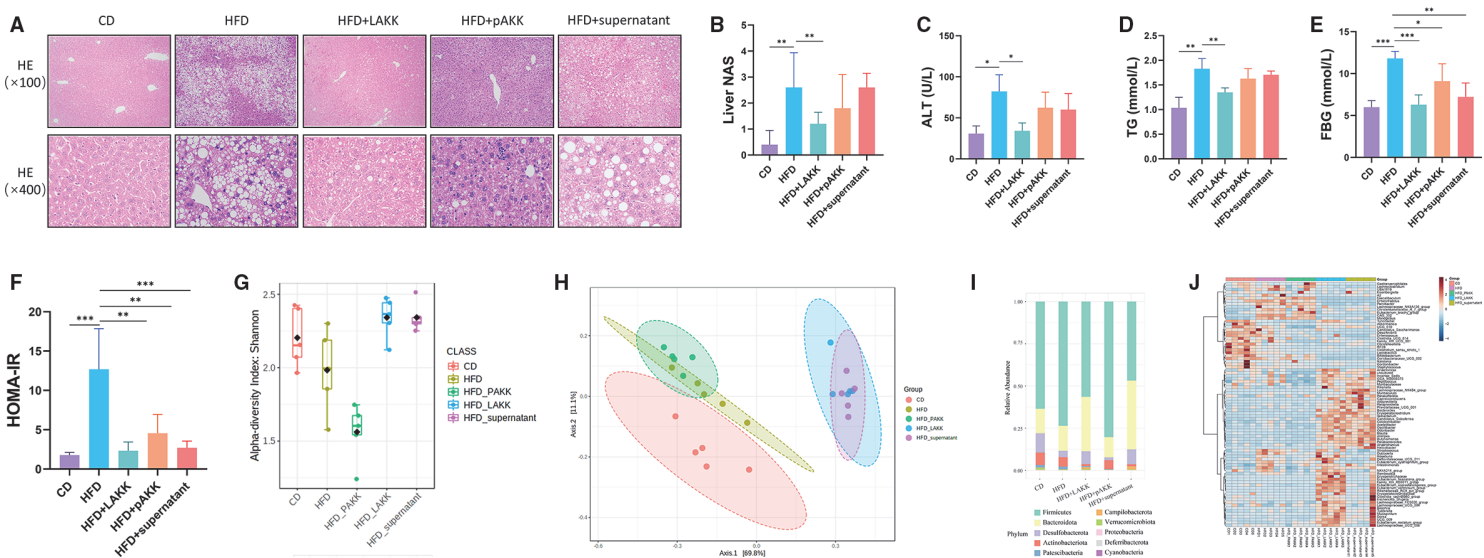